\title{
Impact of Liver Injury in COVID-19 Patients: Single-center Retrospective Cohort Analysis
}

\author{
Jiten Desai*, Upenkumar Patel, Shiva Arjun, Kristen Farraj, Kevin Yeroushalmi, \\ Sandra Gomez Paz, Jaehyuck Im, Andres Castillo, Rajmohan Rammohan and Paul Mustacchia
}

Department of Internal Medicine, Nassau University Medical Center, East Meadow, NY, USA

Citation of this article: Desai J, Patel U, Arjun S, Farraj K, Yeroushalmi K, Paz SG, et al. Impact of liver injury in COVID19 patients: Single-center retrospective cohort analysis. J Clin Transl Hepatol 2020;8(4):476-478. doi: 10.14218/ JCTH.2020.00075.

\section{Introduction}

Coronavirus disease 2019 (COVID-19) has become a serious threat to global public health. Unfortunately, to date, there are no specific vaccines or targeted drugs, and the number of patients with positivity for systemic acute respiratory syndrome-novel coronavirus-2 infection is growing worldwide. ${ }^{1}$ Patients with COVID-19 may be at risk for liver injury, but the mechanism and clinical significance of injury remains unclear. Proposed mechanisms include direct virus-induced insults, immune-mediated damage (due to excessive inflammatory response), and drug-induced injury. COVID-19-related liver dysfunction is now gaining widespread attention; however, liver injury's impact on the outcome of COVID-19 patients is not clearly understood. We have evaluated the impact of liver insults on the hospitalization outcome of COVID-19 patients admitted to a suburban New York safety-net hospital and would like to share our preliminary results in a Letter to the Editor instead of an Original Article for rapid dissemination to the worldwide audience.

In our retrospective, unmatched, single-center analysis, we have identified the first 639 confirmed COVID-19 patients (ages $\geq 18$ years) admitted to our facility from March 2020 to May 2020. Elevated liver-related enzymes [serum alanine aminotransferase (commonly referred to as ALT) $>40 \mathrm{U} / \mathrm{L}$, aspartate aminotransferase (commonly referred to as AST) $>40 \mathrm{U} / \mathrm{L}$, or alkaline phosphatase (commonly referred to as ALP) $>120$ ] were used to stratify patients with or without liver injury. The primary outcome was all-cause in-hospital mortality; other in-patient outcomes, including cardiac arrest, acute respiratory distress syndrome, arrhythmia, shock, and intubation rate, were also measured. The Pearson's chi-square test and Student's $t$-test were used for evaluating categorical

Abbreviations: ACE2, ACE-2 receptors; ALP, alkaline phosphatase; ALT, alanine aminotransferase; aOR, adjusted odds ratio; AST, aspartate aminotransferase; CI, confidence interval; COVID-19, coronavirus disease 2019.

Received: 6 August 2020; Revised: 7 October 2020; Accepted: 8 October 2020 *Correspondence to: Jiten Desai, Department of Internal Medicine, Nassau University Medical Center, 2201 Hempstead Turnpike, East Meadow, NY 11554, USA. Tel: +1-516-296-2253, E-mail: jdesai1@numc.edu and continuous variables, respectively. A two-step hierarchical multivariate regression model was performed to assess the risk of in-patient mortality and other hospitalization outcomes after adjusting for baseline characteristics and comorbidities. All statistical analyses were performed using SPSS ${ }^{\circ}$ Corp. Version 22 (Armonk, NY, USA). This analysis was approved by the Institutional Review Board (IRB) of Nassau Health Care Corporation (NHCC) at Nassau University Medical Center (NUMC), under IRB reference \# 20-277.

Out of the total 639 COVID-19 patients, 476 (74.5\%) [mean age of $58.89 \pm 15.61$ years, $63.0 \%$ male] had evidence of liver injury. COVID-19 liver injury cohorts had statistically significant higher rates of all cause in-patient mortality [35.5\% vs. $22.7 \%$; adjusted odds ratio (aOR): $2.84 ; 95 \%$ confidence interval $(\mathrm{CI}): 1.71-4.71 ; p<0.001)$. COVID-19 liver injury was observed more often in our Hispanic patient population (38.2\%). The COVID-19 liver injury group showed higher risk of other in-patient outcomes, such as cardiac arrest $(26.1 \%$ vs. $14.1 \%$; aOR: $2.65 ; 95 \%$ CI: $1.52-4.59$; $p \leq 0.001)$, requirement of intubation ( $30 \%$ vs. $14.7 \%$; aOR: $2.87 ; 95 \%$ CI: $1.70-4.85 ; p<0.001)$, acute respiratory distress syndrome $(43.1 \%$ vs. $30.7 \%$; aOR: $1.89 ; 95 \% \mathrm{CI}$ : $1.23-2.91 ; p=0.004)$, arrhythmia (5.2\% vs. $0.6 \%$; aOR: $3.16 ; 95 \% \mathrm{CI}: 0.95-10.33 ; p=0.05)$ and shock (15\% vs. $2.8 \%$; aOR: $2.06 ; 95 \% \mathrm{CI}: 1.15-3.70 ; p=0.016)$ compared to COVID-19 patients without evidence of liver injury (Tables 1 and 2).

The infection of liver cells with the systemic acute respiratory syndrome-novel coronavirus-2 (SARS-CoV-2) may directly cause liver dysfunction. Other indirect mechanisms of liver injury are also plausible. Chen et $a .^{2}$ showed that more than one-third of COVID-19 patients have some liver dysfunction; in most cases, patients had mild-to-moderate elevations of ALT or AST levels. Our analysis has shown that approximately every fourth patient presented evidence of liver injury.

It is postulated that SARS-CoV-2 binds to host ACE-2 receptors (ACE2) on target cells to gain entry. Interestingly, ACE2 receptors are also highly expressed within the biliary tree. However, the cholestatic liver disease is not a common feature of COVID-19. ${ }^{3}$ Chau et al. ${ }^{4}$ demonstrated in their study that liver biopsies of SARS patients showed a significant increase in mitotic cells, eosinophils and balloon-like liver cells, which indicated that SARS-CoV-2 might induce liver cell apoptosis and thus lead to liver damage. The study by Tan et al. ${ }^{5}$ showed that SARS-CoV-2 specific protein 7a could induce cell apoptosis in different organs (including lung, kidney, and liver) through the caspase-dependent 
Desai J. et al: Impact of liver injury in Covid-19 patients

Table 1. Baseline characteristics of COVID-19 hospitalizations with vs. without liver injury

\begin{tabular}{|c|c|c|c|}
\hline Variable & With liver injury, $n=476(74.5 \%)$ & Without liver injury, $n=163(25.5 \%)$ & $p *$ \\
\hline \multicolumn{4}{|l|}{ Age in years at admission } \\
\hline Mean age \pm standard deviation & $58.89 \pm 15.61$ & $61.92 \pm 17.32$ & 0.038 \\
\hline Sex & & & 0.041 \\
\hline Male & $63.0 \%$ & $54.0 \%$ & \\
\hline Female & $37.0 \%$ & $46.0 \%$ & \\
\hline Race & & & 0.26 \\
\hline White & $22.1 \%$ & $21.5 \%$ & \\
\hline African American & $24.6 \%$ & $30.7 \%$ & \\
\hline Hispanic & $38.2 \%$ & $30.7 \%$ & \\
\hline Asian or Pacific Islander & $2.9 \%$ & $6.1 \%$ & \\
\hline Other & $11.1 \%$ & $9.8 \%$ & \\
\hline Insurance status & & & 0.23 \\
\hline Uninsured/self-pay & $16.0 \%$ & $16.0 \%$ & \\
\hline Medicare/Medicaid & $40.1 \%$ & $45.4 \%$ & \\
\hline Private insurance & $41.8 \%$ & $34.4 \%$ & \\
\hline Unknown & $1.9 \%$ & $4.3 \%$ & \\
\hline \multicolumn{4}{|l|}{ Comorbidities } \\
\hline Alcohol abuse & $14.9 \%$ & $10.4 \%$ & 0.268 \\
\hline Asthma/COPD & $10.5 \%$ & $11.0 \%$ & 0.713 \\
\hline HIV/AIDS & $1.1 \%$ & $1.8 \%$ & 0.789 \\
\hline Congestive heart failure & $4.8 \%$ & $8.6 \%$ & 0.247 \\
\hline Diabetes mellitus & $34.5 \%$ & $43.6 \%$ & 0.078 \\
\hline OSA/OHS & $1.9 \%$ & $0.6 \%$ & 0.521 \\
\hline Hypertension & $50.6 \%$ & $58.9 \%$ & 0.221 \\
\hline Malignancy & $5.0 \%$ & $3.7 \%$ & 0.321 \\
\hline Chronic kidney disease & $7.6 \%$ & $15.3 \%$ & 0.010 \\
\hline Coronary artery disease & $8.0 \%$ & $14.1 \%$ & 0.05 \\
\hline \multicolumn{4}{|l|}{ In- hospital Outcomes } \\
\hline All cause in-hospital mortality & $35.5 \%$ & $22.7 \%$ & $<0.001$ \\
\hline Cardiac arrest & $26.1 \%$ & $14.1 \%$ & $<0.001$ \\
\hline Intubation & $30 \%$ & $14.7 \%$ & $<0.001$ \\
\hline ARDS & $43.1 \%$ & $30.7 \%$ & 0.004 \\
\hline Arrhythmias & $5.2 \%$ & $0.6 \%$ & 0.05 \\
\hline Shock & $15 \%$ & $2.8 \%$ & 0.016 \\
\hline
\end{tabular}

${ }^{*} p \leq 0.05$ at $95 \%$ confidence interval indicates statistical significance.

Abbreviations: ARDS, acute respiratory response syndrome; COPD, chronic obstructive pulmonary disease; OSA/OHS, obstructive sleep apnea/obesity hypoventilation syndrome; HIV/AIDS, human immunodeficiency virus/acquired immunodeficiency syndrome.

pathway, further confirming the possibility of SARS-CoV-2 directly attacking liver tissues and causing liver damage. In our analysis, COVID-19 patients with liver injury demonstrated nearly a three times higher risk of in-patient mortality and other poor hospital outcomes, including cardiac arrest, acute respiratory distress syndrome, the requirement of intubation, arrhythmia, and shock. Our analysis also showed that male and Hispanic patients were more likely to develop COVID-19associated liver dysfunction than infected female patients and those of other ethnicities. We recommend studies be designed on a large scale, to better understand the sex and race-related difference that our data suggest.

Studies are also needed to assess the outcome of COVID19 patients with preexisting liver disease. Our analysis could not differentiate direct COVID-19-induced hepatotoxicity, drug-induced liver injury, or 'bystander effects' of systemic inflammatory response syndrome caused by the virus, which could be a major limitation of our analysis. Regardless of the mechanisms intricated in the liver injury of patients with COVID-19, worse hospitalization outcomes were noted in our analysis, requiring close monitoring. In the meantime, we 
Desai J. et al: Impact of liver injury in Covid-19 patients

Table 2. Hospitalization outcomes among patients with COVID-19 with liver injury

\begin{tabular}{|c|c|c|c|}
\hline Hospitalization outcome & $\mathrm{aOR}$ & $95 \% \mathrm{CI}$ & $p *$ \\
\hline All cause in-hospital mortality & 2.84 & $1.71-4.71$ & $<0.001$ \\
\hline Cardiac arrest & 2.65 & $1.52-4.59$ & $<0.001$ \\
\hline Intubation & 2.87 & $1.70-4.85$ & $<0.001$ \\
\hline ARDS & 1.89 & $1.23-2.91$ & 0.004 \\
\hline Arrhythmias & 3.16 & $0.95-10.33$ & 0.05 \\
\hline Shock & 2.06 & $1.15-3.70$ & 0.016 \\
\hline
\end{tabular}

${ }^{*} p \leq 0.05$ at $95 \% \mathrm{CI}$ indicates statistical significance.

Abbreviations: aOR, adjusted odds ratio; ARDS, acute respiratory response syndrome; CI, confidence interval.

believe that the front-line medical staff should pay attention to liver-related tests in patients infected with COVID-19. We recommend using appropriate hepatoprotective therapies, especially in patients with preexisting liver disease, to attenuate the potentially deleterious impact of COVID-19related liver dysfunction. ${ }^{6}$

Thus, in our preliminary observations, we noted that COVID-19 patients with liver injury demonstrated nearly a three times higher risk of in-patient mortality and other poor hospitalization outcomes.

\section{Acknowledgments}

The authors are grateful to Daniel J Whitson, Amanda M Silverio, Allen Tsiyer, Raghda Abdelrady and David Gruen in helping in data collection for this analysis.

\section{Funding}

None to declare.

\section{Conflict of interest}

The authors have no conflict of interests related to this publication.

\section{Author contributions}

Conception and design (JD, UP, SA, PM), acquisition of data (JD, $\mathrm{UP}, \mathrm{SA}, \mathrm{KF}, \mathrm{KY}, \mathrm{SGP}, \mathrm{JI}, \mathrm{AC}, \mathrm{RR})$, analysis and interpretation of data (JD, UP, SA, PM), drafting of the manuscript (KF, KY, SGP, JI, AC, RR), critical revision of the manuscript (JD, UP, PM).

\section{References}

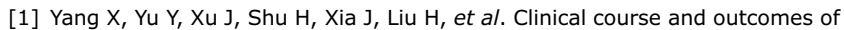
critically ill patients with SARS-CoV-2 pneumonia in Wuhan, China: a singlecentered, retrospective, observational study. Lancet Respir Med 2020;8:475481. doi: 10.1016/S2213-2600(20)30079-5.

[2] Chen N, Zhou M, Dong X, Qu J, Gong F, Han Y, et al. Epidemiological and clinical characteristics of 99 cases of 2019 novel coronavirus pneumonia in Wuhan, China: a descriptive study. Lancet 2020;395:507-513. doi: 10. 1016/S0140-6736(20)30211-7.

[3] Zhou $P$, Yang $X L$, Wang $X G$, Hu B, Zhang $L$, Zhang $W$, et al. A pneumonia outbreak associated with a new coronavirus of probable bat origin. Nature 2020;579:270-273. doi: 10.1038/s41586-020-2012-7.

[4] Chau TN, Lee KC, Yao H, Tsang TY, Chow TC, Yeung YC, et al. SARS-associated viral hepatitis caused by a novel coronavirus: report of three cases. Hepatology 2004;39:302-310. doi: 10.1002/hep.20111.

[5] Tan YJ, Fielding BC, Goh PY, Shen S, Tan TH, Lim SG, et al. Overexpression of $7 a$, a protein specifically encoded by the severe acute respiratory syndrome coronavirus, induces apoptosis via a caspase-dependent pathway. J Virol 2004;78:14043-14047. doi: 10.1128/JVI.78.24.14043-14047.2004.

[6] Cheng ZJ, Shan J. 2019 Novel coronavirus: where we are and what we know. Infection 2020;48:155-163. doi: 10.1007/s15010-020-01401-y. 